\title{
Das antisemitische Unbewusste. Zur politischen Psychologie des Antisemitismus in der Bundesrepublik Deutschland
}

\author{
Wolfram Stender
}

Einer Studie der Anti-Defamation League aus dem Jahr 2014 zufolge gibt es weltweit über eine Milliarde Menschen mit antisemitischen Einstellungsmustern. Im Mittleren Osten und Nordafrika scheint die antijüdische Aversion besonders weit verbreitet. Durchschnittlich 74 Prozent der erwachsenen Bevölkerung stimmen dort den Phantasmen von der unheimlichen Macht, der Geldgier, dem Überlegenheitswahn und der Menschenfeindlichkeit der Juden zu. In Staaten wie Jordanien, Irak oder Algerien sollen es 90 Prozent sein, in der Westbank und im Gazastreifen 93 Prozent. Davon scheint Europa weit entfernt. Mit 4,4 Prozent weist Schweden im Jahr 2014 den niedrigsten Wert auf, Frankreich liegt bei 37 Prozent, Deutschland nimmt mit 27 Prozent einen Mittelplatz ein. Spitzenreiter ist Griechenland mit 69 Prozent, gefolgt von Rumänien mit 47 und Polen mit 45 Prozent. Bemerkenswert ist die Varianz des Phänomens. Der Zustimmungswert bei dem Item „Jews still talk too much about what happened to them in the Holocaust" ist in Westeuropa auffallend hoch - in Deutschland und Österreich liegt er jeweils bei 52 Prozent -, im Mittleren Osten und Nordafrika auffallend niedrig. Hingegen dominieren ökonomische und verschwörungsmythische Stereotype vor allem in Osteuropa. Dem Item ,Jews have too much power in the business world" stimmen in Rumänien 60, in Bulgarien 67 und in Ungarn 69 Prozent der Befragten zu. Spitzenreiter ist auch hier das krisengeschüttelte Griechenland mit 85 Prozent. ${ }^{1}$

Bezieht man die Ergebnisse der ADL-Studie auf die migrationsgesellschaftlichen Realitäten westeuropäischer Länder, scheint sich eine Befürchtung zu bestätigen, die Salomon Korn bereits vor 10 Jahren formulierte: „Der gleichzeitig von Südeuropa vordringende islamistische Antisemitismus und der aus dem Osten Europas in die bisherige Europäische Union einsickernde ,klassische' Antisemitismus werden eine ,Zangenbewegung' vollziehen, die den in Westeuropa vorhandenen sekundären und ,schuldreflexiven' Antisemitismus vermutlich stärken wird. Die Folge wäre womöglich eine Art „Kraftdreieck des Antisemitismus' - eine sich wechselseitig stützende Allianz unterschiedlich ausgeprägter Formen der Judenfeindschaft" ${ }^{2}$. Dafür spricht auch das

1 Alle Zahlen aus Anti-Defamation League (Hrsg.), The ADL Global 100. An Index of Anti-Semitism, 2014, [http://global100.adl.org], eingesehen 25.10.2018. - Zur Kritik an der Studie vgl. David Ranan, Muslimischer Antisemitismus. Eine Gefahr für den gesellschaftlichen Frieden in Deutschland?, Bonn 2018, S. 49ff.

2 Zit. n. Andrei S. Markovits, Amerika, dich hasst sich's besser. Antiamerikanismus und Antisemitismus in Europa, 
Bedrohungsgefühl vieler Jüdinnen und Juden, das in den letzten Jahren deutlich zugenommen zu haben scheint. So äußern 37 Prozent der befragten Jüdinnen und Juden in einer aktuellen Studie aus Deutschland, dass sie Angst vor körperlichen Angriffen haben, 58 Prozent vermeiden aus Sicherheitsgründen bestimmte Stadtteile und 70 Prozent tragen wegen erwarteter Stigmatisierung keine äußerlich erkennbaren Symbole. ${ }^{3}$ So realistisch also die Befürchtung Korns zu sein scheint, sind die Konstellationen des Antisemitismus komplizierter, als er annimmt. Es ist keineswegs nur so, dass sich wechselseitig stützende Allianzen bilden. Die viel zitierte „,neue Unübersichtlichkeit“ des Antisemitismus zeigt sich gerade darin, dass sich im lokalen Raum globalisierter Gesellschaftsverhältnisse unterschiedliche Varianten des Antisemitismus verknüpfen, aber auch in Konfrontation zueinander treten. Konfrontationen entstehen heute immer dann, wenn antisemitisch-proisraelische auf antisemitisch-antiisraelische Gruppen stoßen. Auch dies ließ sich 2014 gut beobachten. Während dschihadistische Islamisten gemeinsam mit rechten und linken Antiimperialisten in Europas Hauptstädten mit Parolen wie „Hamas, Hamas, Juden ins Gas!“ oder „Tod den Zionisten!“ durch die Straßen zogen, erklärten die völkischen Nationalisten aus der Neuen Rechten ihre Solidarität mit Israel, das sie als den wichtigsten Außenposten Europas im Krieg gegen die muslimischen Invasoren betrachten. Es handelt sich hier um ein nur scheinbar paradoxes Phänomen konträrer antisemitischer Wahnvorstellungen. Während radikalislamistische Gruppen propagieren, dass „die Juden“ einen globalen Vernichtungskrieg gegen die Gemeinschaft der muslimischen Gläubigen führen, ist die Neue Rechte Europas davon überzeugt, dass die phantasierte islamische Invasion nur ein weiterer Schritt ist hin zur totalen Vernichtung der europäischen Völker, die die vom ,jüdischen Geist“ (Carl Schmitt) besessenen „Liberalen“ und „Kulturmarxisten“ nach 1945 wieder konsequent betreiben.

In Deutschland ist die Konstellation noch einmal besonders. Aus dem singulären Verbrechenshintergrund dieses Landes ergeben sich politisch-psychologische Komplikationen ganz eigener Art. Dies wird immer dann besonders deutlich, wenn es um das „deutsch-jüdische Verhältnis“, vergangenheitspolitische Fragen oder öffentliche Debatten über Antisemitismus geht. Nach wie vor gilt hier das Gesetz der unmöglichen Tatsache, dass nicht sein kann, was nicht sein darf. Der beliebteste Abwehrtopos der Eliten aus Politik, Wissenschaft, Medienarbeit und Justiz anlässlich antisemitischer Vorfälle lautet, man müsse erst einmal definieren, was Antisemitismus überhaupt sei. Und bei dieser nicht enden wollenden Definitionsarbeit werden dann die unglaublichsten Verdrehungen und Verrenkungen vorgenommen, die schließlich immer wieder darauf hinauslaufen, dass es Antisemitismus vor 1945 gegeben hat, aber heute nicht mehr gibt. Er sei, so wird behauptet, in einem kollektiven Lernprozess überwunden worden, was

Hamburg 2008, S. 208.

${ }_{3}^{3}$ Bericht des Unabhängigen Expertenkreises Antisemitismus, 7.4.2017, Deutscher Bundestag, Drucksache 18/11970, S. 102ff., [http://dip21.bundestag.de/dip21/btd/18/119/1811970.pdf], eingesehen 25.10.2018. 
sich unter anderem in der prächtigen Erinnerungskultur dieses Landes zeige. Wenn überhaupt, dann trete Antisemitismus heute nur noch als Gefahr auf, die von außen das anti-antisemitisch geläuterte Gemeinwesen bedrohe - in Gestalt des Rechts- oder Linksextremismus oder als Importprodukt von meistens als „muslimisch“ markierten Migranten und Migrantinnen.

Dass das Narrativ vom anti-antisemitischen Lernprozess auf einer Realitätsabspaltung beruht, die zum Kern jener in Deutschland besonders ausgeprägten Form des Antisemitismus gehört, die als ,sekundär“ bezeichnet wird, ist eine These, die im Folgenden genauer begründet werden soll. ${ }^{4}$ Die nationalstaatliche Legende erzählt nur die halbe Wahrheit, die positive Geschichte der erfolgreichen Eindämmung des politischen Antisemitismus, nicht aber die verborgene, unterirdische, aber darum umso wirkungsmächtigere Geschichte der Tradierung der antisemitischen Erbschaft nach Auschwitz. Kennt man diese Geschichte nicht, bleiben einem einige Besonderheiten in der politischen Kultur der Bundesrepublik Deutschland völlig verschlossen.

\section{Sekundärer Antisemitismus und philosemitisches Syndrom: die Nachkriegszeit}

Aus Anlass des sogenannten Historikerstreits in den 1980er-Jahren - also des Versuchs mehrerer nationalkonservativer Historiker, qua „Geschichtsentsorgung“ (Jürgen Habermas) eine positive deutsch-nationale Identität wiederherzustellen - beschrieb Dan Diner „das Verhältnis von Deutschen und Juden nach Auschwitz“ als „negative Symbiose“. Er prognostizierte, dass mit zunehmender zeitlicher Distanz zum Ereignis dieses Verhältnis nicht einfacher, sondern schwieriger werde. ${ }^{5}$ Den Grund dafür sah er darin, dass Auschwitz für beide - nicht-jüdische Deutsche wie Juden - Ausgangspunkt ihres Selbstverständnisses geworden sei, allerdings in gegensätzlicher Perspektive. Leben im Schatten des Zivilisationsbruchs sei für das „Kollektiv der Opfer“ unerträglich, für das „Kollektiv der Täter“ unbehaglich. Während die Täter, Mittäter und Mitläufer und deren Nachgeborenen der Erinnerung ausweichen und Wege der Entlastung suchen, empfinden dies die überlebenden Opfer und deren Kinder als Anschlag auf das kollektive Gedächtnis. Ob aus der Psychodynamik des Unbehagens an der Vergangenheit ein neuer Antisemitismus werden könne, hielt Diner für eine plausible Fragestellung: „Und solche Manöver des Ausweichens [auf Seiten der Täter und ihrer Nachgeborenen; Anm. WS] wiederum vermögen angesichts ständigen jüdischen Gemahnens an das Grauen sich in

${ }^{4}$ Ich werde mich im Folgenden allein auf die Geschichte des Antisemitismus in der BRD beziehen, zur besonderen Geschichte der DDR siehe Thomas Haury, Antisemitismus von links. Kommunistische Ideologie, Nationalismus und Antizionismus in der frühen DDR, Hamburg 2002.

${ }^{5}$ Dan Diner, Negative Symbiose. Deutsche und Juden nach Auschwitz, in: Ders. (Hrsg.), Ist der Nationalsozialismus Geschichte? Zu Historisierung und Historikerstreit, Frankfurt a. M. 1987, S. 185-197, hier S. 195. 
blinde Wut zu steigern - Antisemitismus wegen Auschwitz?“.6 Die These eines Antisemitismus ,nicht trot\%, sondern wegen Auschwitz ${ }^{67}$ findet sich zeitgleich auch bei Henryk Broder. Mit Bezug auf das Theaterstück „Der Müll, die Stadt und der Tod“8 von Rainer Werner Fassbinder, das wegen seiner antisemitischen Inhalte in den 1980erJahren heftige Kontroversen auslöste, beschreibt er, wie aus unbewusster Beschämungsund Bestrafungsangst ein antisemitischer Hass entsteht: ,Jeder lebende und überlebende Jude ist Zeuge und Vorwurf zugleich. Wenn ,es ' in dem Antisemiten Hans von Gluck in Fassbinders Stück laut denkt: ,Und schuld ist der Jud, weil er uns schuldig macht, denn er ist da. Wär er geblieben, wo er herkam, oder hätten sie ihn vergast, ich könnte heute besser schlafen. Sie haben vergessen, ihn zu vergasen...', dann kommt in solchen Sätzen die Wut der Täter auf die Opfer zum Ausdruck, deren Immer-noch-da-Sein eine kaum erträgliche Provokation ist".?

Was Diner und Broder in den 1980er-Jahren beobachteten, war keine neue Entdeckung. Schon drei Jahrzehnte vorher war das Frankfurter Institut für Sozialforschung in seinen empirischen Studien auf den neuen, sekundären Antisemitismus gestoßen. Der Begriff findet sich zum ersten $\mathrm{Mal}$ in der kleinen empirischen Studie „Reaktionen auf die antisemitische Welle im Winter 1959/1960“von Peter Schönbach. Anlass für die Studie waren antisemitische Schmierereien an vielen Orten in der Bundesrepublik, die das nach 1945 mühsam errichtete „öffentliche Tabu des Antisemitismus“10 konterkarierten. Schönbach interessierten aber nicht die antisemitischen Vorfälle selbst, sondern die Reaktionen in der Bevölkerung, die er am Beispiel der Stadt Frankfurt untersuchte. Als besonders wertvoll erwies sich, dass die Forschungsergebnisse mit einer zeitgleich in den USA von Johan Galtung durchgeführten Studie ${ }^{11}$ verglichen werden konnten. Auch in den USA war es im Frühjahr 1960 zu einer Welle antisemitischer Handlungen gekommen, die nach Umfang und Verlauf mit der in der Bundesrepublik auffällig übereinstimmte. Während aber in der Frankfurter Befragung die als antisemitisch Eingestuften (ca. 16 Prozent) die Ereignisse mehrheitlich bagatellisierten und als „unpolitische Streiche von Halbstarken und dummen Jungen“ abtaten, war in den USA das genaue Gegenteil der Fall: die Vorfälle wurden als „politisch sehr bedeutsam“ aufgewertet. Mit der Beobachtung dieser Diskrepanz in den Reaktionen der Antisemiten waren die Forscher und Forscherinnen auf einen bedeutsamen Sachverhalt gestoßen: Je nach politisch-psychologischem Kontext, so lautete der Befund, fallen die antisemitischen Äußerungsformen unterschiedlich aus.

Das Besondere des bundesrepublikanischen Kontextes lag darin, dass sich der demokratische Neuanfang in der erfolgreichen Bekämpfung des Antisemitismus zu

\footnotetext{
${ }^{6}$ Diner, Negative Symbiose, S. 186.

${ }^{7}$ Henryk M. Broder, Der ewige Antisemit. Über Sinn und Funktion eines beständigen Gefühls, Berlin 2005, S. 37.

${ }^{8}$ Rainer Werner Fassbinder, Der Müll, die Stadt und der Tod. Nur eine Scheibe Brot, Frankfurt a. M. 1998.

${ }^{9}$ Broder, Der ewige Antisemit, S. $37 \mathrm{f}$.

${ }^{10}$ Peter Schönbach, Reaktionen auf die antisemitische Welle im Winter 1959/1960, Frankfurt a. M. 1961, S. 5.

${ }^{11}$ Johan Galtung, Anti-Semitism in Making. A Study of American High School Students, Oslo 1960.
} 
beweisen hatte. Antisemitismus durfte es offiziell nicht mehr geben, aber natürlich gab es ihn noch überall. Daraus entstand eine eigentümliche Doppelstruktur aus öffentlichem Tabu und antisemitischer Latenz. ${ }^{12}$ Konnten antisemitische Stereotype öffentlich nicht mehr geäußert werden, lebte das antijüdische Ressentiment auf der Ebene der Alltagskultur als nicht-öffentliche Meinung fort. Diese paradoxe Gleichzeitigkeit von öffentlicher Tabuisierung und alltagskultureller Reproduktion des Antisemitismus stand auch im Zentrum der Studie von 1961. Schönbach beobachtete, dass die Konfrontation mit den nationalsozialistischen Verbrechen bei gleichzeitiger offizieller Ächtung des weltanschaulichen Antisemitismus massive Schuldabwehr-Mechanismen bei vielen ehemaligen NS-Volksgenossen mobilisierte. In dem prekären Nebeneinander von Rechtfertigungs- und Leugnungsreaktionen der Befragten wurde eine „psychologische Aporie" sichtbar, in der sich Antisemiten in Deutschland nach 1945 befanden. Exakt auf diese Aporie bezog sich der Begriff des „,sekundären Antisemitismus“, mit dem die Studie zugleich eine generationengeschichtliche Perspektive für die Antisemitismusforschung eröffnete. ${ }^{13}$

Man habe vom Geschehenen damals nichts gewusst; schuld sei allein eine kleine Clique gewesen; andere hätten sich auch die Finger schmutzig gemacht; es müsse jetzt auch mal Schluss sein; die Juden seien letztlich an allem selber schuld; und wenn sie jetzt ständig Forderungen nach Entschädigung stellten, bräuchten sie sich nicht zu wundern, dass der Antisemitismus wiederauflebe: in solchen und ähnlichen Abwehräußerungen, vielfach dokumentiert in der großen empirischen Studie „Schuld und Abwehr"14 von Theodor W. Adorno aus dem Jahr 1955, trat das ideologische Syndrom des sekundären Antisemitismus deutlich hervor. Es ermöglichte dem Antisemiten, den Massenmord an den europäischen Juden zu relativieren, zugleich jede Mitschuld an ihm zu leugnen und in dieser Verleugnung antisemitische Stereotype zu reproduzieren. Die Attraktivität dieser Form des Antisemitismus lag darin, dass sie für die psychologische Aporie der ehemaligen NS-Volksgenossen unter postnazistischen politischen Rahmenbedingungen eine Lösung anbot: Man konnte sich gegenüber den neuen politischen Mächten konform geben, ohne die narzisstische Besetzung der eigenen (Familien-) Geschichte aufgeben zu müssen. Dem kam nicht nur die von Anfang an weit verbreitete Schlussstrichhaltung, sondern auch die von Teilen der politischen Führung wie ein Mantra rezitierte Beschwörungsformel entgegen, dass es Antisemitismus zwar vor 1945 gegeben habe, aber jetzt nicht mehr gebe: „Der Bazillus des Antisemitismus ist im deutschen

12 Werner Bergmann/Rainer Erb, Kommunikationslatenz, Moral und öffentliche Meinung. Theoretische Überlegungen zum Antisemitismus in der Bundesrepublik Deutschland, in: KZfSS 38 (1986), Heft 2, S. 223-246. Der Begriff des antisemitischen Unbewussten, der im Folgenden für den Antisemitismus im postnazistischen Deutschland vorgeschlagen wird, geht über die von Bergmann und Erb systemtheoretisch gefasste Kommunikationslatenz hinaus und umfasst auch Elemente einer psychoanalytisch-sozialpsychologischen Bewusstseinslatenz.

${ }^{13}$ Schönbach, Reaktionen auf die antisemitische Welle, S. 80.

14 Theodor W. Adorno, Schuld und Abwehr [1955], in: Ders., Gesammelte Schriften, Bd. 9.2, Frankfurt a. M. 1997, S. $121-324$. 
Volkskörper selbst nicht mehr virulent ", 15 formulierte im Jahr 1960 - also zeitgleich zur Schönbach-Studie - als einer von vielen der Staatsrechtler und prominente SPD-Politiker Carlo Schmid und bestätigte damit nur die ohnehin von der Mehrheit getragene Weigerung, sich in irgendeiner Weise mit dem fortbestehenden Problem des Antisemitismus auseinanderzusetzen.

Die Mechanismen des sekundären Antisemitismus hat Adorno in „Schuld und Abwehr“ und weiteren Aufsätzen aus den 1950er und 1960er-Jahren ${ }^{16}$ sehr genau analysiert. Deutlich erkannte er das „Neurotische“ vieler Deutscher im Verhältnis zur NSVergangenheit wie auch im Verhältnis zu noch lebenden Jüdinnen und Juden: „Gesten der Verteidigung dort, wo man nicht angegriffen ist; heftige Affekte an Stellen, die sie real kaum rechtfertigen; Mangel an Affekt gegenüber dem Ernstesten; nicht selten auch einfach Verdrängung des Gewussten oder halb Gewussten. So sind wir im Gruppenexperiment des Instituts für Sozialforschung häufig darauf gestoßen, dass bei Erinnerungen an Deportation und Massenmord mildernde Ausdrücke, euphemistische Umschreibungen gewählt werden oder ein Hohlraum der Rede sich darum bildet“". ${ }^{17}$ Bildet die Schuldabwehraggression gegen Jüdinnen und Juden den Kern des sekundären Antisemitismus in den ersten beiden Jahrzehnten nach Auschwitz, so sind es für Adorno vor allem vier Elemente, die die politische Psychologie dieser Form eines postnazistischen Antisemitismus bestimmen: das von außen erzwungene Kommunikationsverbot für antisemitische Äußerungen - Adorno spricht von den „offiziellen Tabus, die [...] über dem Antisemitismus liegen“18 _, das Fortbestehen nationaler Identifikationen und kollektiver Größenphantasien bei vielen ehemaligen NSVolksgenossen bei gleichzeitiger faktischer Schädigung des kollektiven Narzissmus, ${ }^{19}$

15 Zit. n. Werner Bergmann, Antisemitismus in öffentlichen Konflikten. Kollektives Lernen in der Bundesrepublik 1949-1989, Frankfurt a. M. 1997, S. 237. - Man könnte auch Adenauer, Kohl, Schmidt oder irgendeinen anderen bundesdeutschen Spitzenpolitiker nennen. In der Tendenz, Antisemitismus in der Gegenwart zu leugnen, bestand ein weitgehend parteiübergreifender Konsens.

16 Siehe u. a. Theodor W. Adorno, Was bedeutet: Aufarbeitung der Vergangenheit [1959], in: Ders., Gesammelte Schriften Bd. 10.2, Frankfurt a. M. 1997, S. 555-572; Ders., Meinung Wahn Gesellschaft [1961], in: Ders., Gesammelte Schriften, Bd. 10.2, Frankfurt a. M. 1997, S. 573-594; Ders., Zur Bekämpfung des Antisemitismus [1962], in: Ders., Gesammelte Schriften, Bd. 20.1, Frankfurt a. M. 1997, S. 360-383.

17 Adorno, Was bedeutet: Aufarbeitung, S. 556. Dass das „Neurotische“ im Verhältnis zu Jüdinnen und Juden sich bis heute fortsetzt, weiß jeder und jede, der/die einmal in Konflikte um antisemitische Vorfälle involviert war. Gesten der Verteidigung, wo man nicht angegriffen wurde, heftige Affekte an Stellen, die sie real kaum rechtfertigen - ,es“ denkt in den Kindern und Enkeln der NS-Volksgenossen immer sehr heftig, wenn sie mit Juden und Jüdinnen in Konflikt geraten. Beim Skandal um ein antisemitisches Seminar an der Hochschule für angewandte Wissenschaft und Kunst Hildesheim im Jahr 2016 konnte man dies exemplarisch an den Äußerungen der Hochschulpräsidentin beobachten, die meinte, der Öffentlichkeit mitteilen zu müssen, dass sie ihren Kindern jüdische Vornamen gegeben habe, sich aber nicht „von einflussreichen Kreisen“ des Antisemitismus bezichtigen lasse. Wozu habe ich meinen Kindern jüdische Vornamen gegeben, dass ich mich jetzt von den Juden fertig machen lassen muss!, so denkt „es“ in ihr.

18 Adorno, Zur Bekämpfung des Antisemitismus, S. 361.

${ }^{19}$ Die These Adornos, dass alle Indikatoren, die auf ein Zerbrechen der kollektiven Identifikationen hätten hindeuten können, nach 1945 fehlten - weder waren Anflüge von Panik noch von Depressionen bei den ehemaligen NSVolksgenossen zu erkennen - und deshalb davon auszugehen sei, dass diese ,insgeheim, unbewusst schwelend und darum besonders mächtig“ (Adorno, Was bedeutet: Aufarbeitung, S. 564) fortbestanden, trifft sich mit den 
diffuse Gefühle von Schuld und Scham, die durch die Erinnerung an Auschwitz hervorgerufen werden, und schließlich die tradierte antijüdische Aversion selbst, die kulturell so tief verankert ist, dass sie nach 1945 unmöglich ihre Wirkung verloren haben konnte. Im Kraftfeld dieser widerstrebenden politisch-psychologischen Tatsachen konstituierte sich ein antisemitisches Unbewusstes ${ }^{20}$ in der politischen Kultur der Bundesrepublik, dessen generationenübergreifende Virulenz bis heute nachweisbar ist ${ }^{21}$ und das in dem „Neurotischen“ (Adorno) des deutsch-jüdischen Verhältnisses immer wieder zum Ausdruck drängt. Je umfassender das antisemitische Kommunikationsverbot etabliert war, umso erfinderischer wurden die Wege, um das Verbotene - das antisemitische Ressentiment - unerkannt in die Sprache und das Handeln zu schmuggeln - unerkannt durchaus, wenn auch keineswegs immer, auch für die antisemitischen Subjekte selbst. Nur in Konflikten - Augenblicken der Wahrheit - brach es mitunter offen und unwillkürlich aus den zum Verbot Gezwungenen heraus, die Geschichte der Bundesrepublik ist reich an Beispielen davon. ${ }^{22}$ Dabei ähnelten die Äußerungsformen des antisemitischen Unbewussten, das man später paradox auch als „Antisemitismus ohne Antisemiten“23 umschrieb, in ihrer inneren Struktur jenen Kompromiss- und Ersatzbildungen, die Freud ja nicht nur für den Traum, sondern auch für die Psychopathologie des Alltagslebens konstatiert hatte. ${ }^{24}$

Beobachtungen Hannah Arendts (Hannah Arendt, Besuch in Deutschland [1950], in: dies., Zur Zeit. Politische Essays, Berlin 1986, S. 43-70.) ebenso wie mit den Überlegungen der Mitscherlichs (Alexander Mitscherlich/Margarete Mitscherlich, Die Unfähigkeit zu trauern. Grundlagen kollektiven Verhaltens, München 1985 [1967].).

20 Es muss zwischen dem Unbewussten des Antisemiten und dem antisemitischen Unbewussten unterschieden werden. Die psychoanalytische Theorie kann die unbewussten Abwehrmechanismen erklären, die der antijüdischen Aversion zugrunde liegen. Fenichel hat diese als konformistische Rebellion beschrieben: „Im Unbewussten der Antisemiten verkörpern die Juden gleichzeitig das, wogegen sie gern rebellieren möchten, und die rebellische Tendenz in ihnen selbst“. (Otto Fenichel, Elemente einer psychoanalytischen Theorie des Antisemitismus, in: Ernst Simmel (Hrsg.), Antisemitismus, Frankfurt a. M. 1993 [engl. 1946], S. 35-57, hier S. 45.) - Das antisemitische Unbewusste hingegen besteht in der Unbewusstmachung der antijüdischen Aversion selbst. Es entsteht überall dort, wo die Regeln der politischen Kultur die antisemitische Kommunikation in die Latenz zwingen. Man hat es hier also mit einer doppelten Unbewusstheit zu tun: Der Antisemit täuscht sich über die unbewussten Motive seiner antijüdischen Aversion; der postnazistische Antisemit, der kein Antisemit mehr sein will, täuscht sich über seine unbewusste antijüdische Aversion, die seiner Israelobsession zugrunde liegt.

${ }^{21}$ Hier erweist sich einmal mehr der besondere Wert der Psychoanalyse für die Antisemitismusforschung, die am Material der analytischen Therapie den Nachweis eines antisemitischen Unbewussten erbracht hat. (Siehe u. a. Anna Maria Jokl, Zwei Fälle zum Thema „Bewältigung der Vergangenheit“, Frankfurt a. M. 1997; Ilka Quindeau, Schuldabwehr und nationale Identität - Psychologische Funktionen des Antisemitismus, in: Matthias Brosch u. a. (Hrsg.), Exklusive Solidarität. Linker Antisemitismus in Deutschland, Berlin 2007, S. 157-164.)

22 Siehe u. a. Bergmann, Antisemitismus in öffentlichen Konflikten; Lars Rensmann, Demokratie und Judenbild. Antisemitismus in der politischen Kultur der Bundesrepublik Deutschland, Wiesbaden 2005, S. 334ff.

23 „Er ist ein ,Antisemitismus ohne Antisemiten‘. Dieser Begriff bezeichnet ein Massenvorurteil ohne Legitimation, ohne öffentliches Subjekt, ohne propagandistische Träger, ohne das Selbstbewusstsein und das Selbstverständnis einer Ideologie [...]. Darüber hinaus ist es als Massenvorurteil durch kulturelle Verfestigung ins, kollektive Unbewusste abgesunken; es wird häufig ohne Absicht und Bewusstsein im alltäglichen Sprachgebrauch reproduziert, ,hinter dem Rücken der Subjekte, über ,Sprachzerstörung' durch bewusstlose Individuen hindurch wirkend.“ (Bernd Marin, Antisemitismus ohne Antisemiten. Autoritäre Vorurteile und Feindbilder, Wien 2000, S. 112.)

24 Sigmund Freud, Zur Psychopathologie des Alltagslebens, in: Ders., Gesammelte Werke. Vierter Band, Frankfurt a. M. 1999. 
Adorno erläutert die Mechanismen antisemitischer Latenz an verschiedenen Formvarianten. Die gängigste Form bestand in der Kryptisierung des Antisemitismus. Im „Krypto-Antisemitismus“,25 wie Adorno es nennt, wird der Judenhass nicht aufgegeben, sondern insgeheim, im Verborgenen fortgesetzt. Der Antisemit verhält sich in der Öffentlichkeit politisch korrekt, verbreitet aber im nicht-öffentlichen Raum weiter seine antisemitischen Ansichten und gibt sich als Widerständler gegen die angeblich von außen aufgezwungene Meinungsdiktatur: „Man darf ja gegen Juden heute nichts sagen“, ${ }^{26}$ lautete ein häufig zu hörender Satz, dessen antisemitischen Gehalt Adorno analysiert: „Es wird sozusagen gerade aus dem öffentlichen Tabu über dem Antisemitismus ein Argument für den Antisemitismus gemacht: wenn man nichts gegen die Juden sagen darf, dann - so läuft die assoziative Logik weiter - sei an dem, was man gegen sie sagen könnte, auch schon etwas daran. Wirksam ist hier ein Projektionsmechanismus: dass die, welche die Verfolger waren und es potentiell heute noch sind, sich aufspielen, als wären sie die Verfolgten. " ${ }^{27}$ Eine zweite Variante bestand darin, dass die Erinnerung an Auschwitz den Juden zum Vorwurf gemacht wurde und in diesem Vorwurf antisemitische Stereotype reproduziert wurden. Typisch dafür waren neben der Schlussstrich-Rhetorik und der Auschwitz-Dresden-Gleichsetzung („die Bombennächte von Dresden waren für uns ja mindestens genauso schlimm wie Auschwitz für die Juden“"28) die Unterstellung, die Juden würden ihren Status als Opfer der NS-Zeit ausnutzen, ${ }^{29}$ um sich finanzielle Vorteile zu verschaffen. Die Relativierung, Minimierung und Bagatellisierung von Auschwitz und die Selbststilisierung der Täter als Opfer stehen im Mittelpunkt dieser Form der Abwehr. In einer dritten Variante schließlich wurde ,der Jude’ ,zu dem Feind, den man lieben muss". ${ }^{30}$ Auch hier ist das Versprechen auf Gewalt, das sich gegen Juden richtet, gut verhüllt: „Auch der sogenannten positiven Stereotypenbildung wäre entgegenzuwirken, hinter der die negative Stereotypie dicht lauert. Sagt einer: ,Die Juden sind alle so gescheit', dann ist er, auch wenn er es lobend sagt, schon nahe bei ,nun ja, und deshalb wollen sie uns betrügen"“. 31

In einer umfassenden Studie hat Frank Stern die dritte Variante der unbewussten Schuldentlastung als „philosemitisches Syndrom“32 analysiert. Seine These lautet, dass Philosemitismus nicht einfach mechanisch als ein auf den Kopf gestellter Antisemitismus zu interpretieren sei. Der philosemitische Habitus, der sich nach 1945 in den Westzonen herausbildete und später zu einem festen Bestandteil der politischen Kultur der

\footnotetext{
25 Adorno, Zur Bekämpfung des Antisemitismus, S. 361.

${ }^{26}$ Heute wird das Wort „Jude“ durch „Israel“ ersetzt, was an dem antisemitischen Gehalt nichts ändert: „Man darf ja gegen Israel heute nichts sagen“.

27 Adorno, Zur Bekämpfung des Antisemitismus, S. 368.

${ }_{28}$ Die Rechte spricht heute vom „Dresdener Bombenholocaust“.

${ }^{29}$ Adorno, Zur Bekämpfung des Antisemitismus, S. 368.

${ }^{30}$ Hermann Greive, Geschichte des modernen Antisemitismus in Deutschland, Darmstadt 1988, S. 184.

31 Adorno, Zur Bekämpfung des Antisemitismus, S. 378.

32 Frank Stern, Im Anfang war Auschwitz. Antisemitismus und Philosemitismus im deutschen Nachkrieg, Gerlingen 1991, S. 351.
} 
Bundesrepublik wurde, habe vielmehr von Anbeginn eine Doppelfunktion gehabt, in der das Verhältnis von Kontinuität und Diskontinuität im deutschen Nachkrieg zum Ausdruck komme. Im Spannungsfeld von offiziellem Antisemitismusverbot, auf dessen Einhaltung die Siegermächte mit Nachdruck pochten, und tiefsitzenden antisemitischen Ressentiments vollzog sich in einem statistisch nicht irrelevanten Teil der deutschen Bevölkerung ein ambivalenter sozialpsychologischer Prozess, in dem es zu einer Metamorphose von antisemitischen in philosemitische Stereotype kam. ${ }^{33}$ Nach Stern war dies keineswegs nur Teil der individuellen Anpassung an die neuen Machtverhältnisse, sondern hatte auch eine „kathartische Funktion“.34 Die philosemitische Metamorphose des Antisemitismus schuf Distanz zum antisemitischen Konsens der NS-Gesellschaft und damit auch zur eigenen Verstrickung in diesen. Stern betont die pragmatische Tendenz, sieht aber auch die irrational-unbewusste Dimension in diesem Verwandlungsprozess. Deutsche begannen ihren neugeborenen Kindern jüdische Vornamen zu geben, suchten nach jüdischen Vorfahren, (er)fanden jüdische Freunde oder Nachbarn, schufen sich also ein projüdisches Phantasma, nicht nur weil sie sich dadurch Vorteile in der neuen Zeit erhofften, sondern auch weil ihnen dies seelische Entlastung brachte. Und genau aus diesem Grund drehten sie auch die antisemitischen Stereotype um, ohne sich je mit ihrem eigenen Antisemitismus auch nur eine Sekunde auseinandergesetzt zu haben. Die Entstehung des Topos vom jüdischen Beitrag zur deutschen Kultur, der bis heute nachhallt, fällt in diese Zeit: „Das in wahrem Crescendo erklingende Lob des jüdischen Beitrags zu deutscher Kultur, Wissenschaft und Wirtschaft wird zu einem festen Topos in der politischen Kultur. Dies traf gleichermaßen zu auf die Ebene sozialer Beziehungen. Mit einem Juden befreundet zu sein, oder derartige Beziehungen aus der Erinnerung zu rekonstruieren, rückte den Betreffenden quasi automatisch an die Seite der Sieger, ließ ihn vermeintlich zu den ehemaligen NSParteigenossen auf Distanz gehen. Die Haltung zu Juden nahm zunehmend den Charakter eines unterschiedslos, ja stereotyp, alles Jüdische positiv wertenden gesellschaftlichen Phänomens an. Und was nicht in dieses überhöhte Bild vom Juden, von jüdischer Eigenart und jüdischem Beitrag passte, fiel schlicht unter Amnesie“. ${ }^{35}$ Eine zweite, in der Gründungsphase der Bundesrepublik zunehmend wichtiger werdende Funktion hatte der Philosemitismus auf der politischen Ebene. Hier diente er der

33 Auch das Frankfurter Institut für Sozialforschung war in dem im Winter 1950/51 durchgeführten „Gruppenexperiment“ - einer Untersuchung zum politischen Bewusstsein der westdeutschen Bevölkerung in 121 Gruppendiskussionen mit insgesamt 1.635 Personen - auf das Phänomen des Philosemitismus gestoßen. Es zeigte sich, dass 18 Prozent der Interviewten philosemitische Positionen einnahmen. Auch Pollock, der die Untersuchung leitete, und seinen Mitarbeitern entging nicht, dass die projüdischen Äußerungen zum Teil große Strukturähnlichkeit mit den antisemitischen Stereotypen aufwiesen und deshalb keineswegs generell positiv zu bewerten seien. (Friedrich Pollock, Gruppenexperiment. Ein Studienbericht (Frankfurter Beiträge zur Soziologie 2), Frankfurt a. M. 1955, S. 128.) 34 Frank Stern, Philosemitismus statt Antisemitismus. Entstehung und Funktion einer neuen Ideologie in Westdeutschland, in: Wolfgang Benz (Hrsg.), Zwischen Antisemitismus und Philosemitismus. Juden in der Bundesrepublik, Berlin 1991, S. 47-61, hier S. 52.

35 Stern, Im Anfang war Auschwitz, S. 16. 
moralischen Legitimierung der westdeutschen Demokratie. Er wurde als Symbol für die Glaubwürdigkeit des demokratischen Erneuerungswillens der Bundesrepublik politisch funktionalisiert.

Philosemitismus ist bis heute ein wichtiges Element in der politischen Kultur Deutschlands geblieben und gilt als „unausgesprochene Staatsdoktrin“36. Auf der Alltagsebene hatte diese Schuldentlastungsform ihre große Zeit in den ersten beiden Jahrzehnten des Nachkriegs. Ihr Niedergang setzte in den 1960er-Jahren ein. Der Wendepunkt lässt sich ziemlich genau bestimmen. Es war der Sechstagekrieg 1967, in dem sich Israel gegen eine Übermacht arabischer Armeen behauptete. Mit ihm veränderte sich der Blick auf Israel und in der Folge zeigte sich, wie rasch die Metamorphose umgekehrt und aus dem Philosemitismus wieder ein Antisemitismus werden kann - in den Worten Frank Sterns: „In gesellschaftlichen Konfrontationen hat der Philosemitismus keinen Bestand: denn in ihm werden die Juden [...] romantisiert, monumentalisiert, respektabel neutralisiert und damit kulturell erneut stigmatisiert und ausgegrenzt. Auch die vermeintlich positive Umkehrung des Antisemitismus macht die Juden zu Fremden; nur dass der gelbe Fleck jetzt geschönt ist, freundlich glänzt" “ ${ }^{37}$

\section{Israelfeinde: der „ehrbare“ Antisemitismus von links in den 1970er und 1980er-Jahren}

Niemand hat so früh und so treffend die antisemitische Kehrseite des Antizionismus auf den Begriff gebracht wie Jean Améry in dem Zeit-Artikel „Der ehrbare Antisemitismus“ von 1969: „Fest steht: der Antisemitismus, enthalten im Anti-Israelismus oder AntiZionismus wie das Gewitter in der Wolke, ist wiederum ehrbar. Er kann ordinär reden, dann heißt das, Verbrecherstaat Israel'. Er kann es auf manierlichere Art machen und vom ,Brückenkopf des Imperialismus' sprechen, dabei so nebstbei allenfalls in bedauerndem Tonfall hinweisen auf die missverstandene Solidarität, die so ziemlich alle Juden, von einigen löblichen Ausnahmen abgesehen, an den Zwergstaat bindet, und kann es empörend finden, dass der Pariser Baron Rothschild die Israel-Spenden der jüdische Bevölkerung Frankreichs als eine Steuer einfordert“.38 Der Sechstagekrieg von 1967 wirkte in Westdeutschland wie ein Fluoreszenzindikator, der blitzartig die antisemitischen Erbschaften auch dort sichtbar machte, wo man sie nicht unbedingt vermutete. Während in der westorientierten, konservativen „Mitte“ der Gesellschaft

36 Wolfgang Benz, Zur Geschichte der organisierten Abwehr des Antisemitismus, in: Jabrbuch für Antisemitismusforschung 20 (2011), S. 15-35, hier S. 17.

${ }^{37}$ Frank Stern, Philosemitismus in Deutschland. Die kulturelle Ambivalenz der Bilder von Juden, in: Heike Catrin Bala/Christian Scholz (Hrsg.), Deutsch-jüdisches Verhältnis? Fragen, Betrachtungen, Analysen, Essen 1997, S. 73-87, hier S. 84 .

38 Jean Améry, Der ehrbare Antisemitismus. Die Barrikade vereint mit dem Spießer-Stammtisch gegen den Staat der Juden, in: Die Zeit, Nr. 30, 25.7.1969. 
zunächst nach bewährter philosemitischer Schuldentlastungsmanier Israels militärische Schlagkraft bejubelt wurde und Vergleiche zwischen Wüstenfuchs Rommel und Mosche Dajan gezogen wurden, vollzog der Sozialistische Deutsche Studentenverbund (SDS) als wichtigste Organisation der außerparlamentarischen Opposition bereits im September 1967 eine deutliche Abkehr vom bis dahin pro-israelischen Konsens der bundesdeutschen Linken. ${ }^{39}$ Die sich danach - begleitet von innerlinken Konflikten herausbildende antizionistische Weltanschauung wurde für zwei Jahrzehnte zum Markenzeichen großer Teile der radikalen Linken, reichte aber auch bis weit in das Alternativmilieu und die Partei „Die Grünen“, die sich 1980 gründete. ${ }^{40}$

Der linke Antizionismus in Westdeutschland entstand im Schnittfeld verschiedener politischer Kraftlinien, zu der nicht zuletzt die massive antikommunistische und mit deutlich antisemitischen Untertönen durchsetzte Hetze des dezidiert pro-israelischen Springer-Konzerns und seiner Medien gegen die Studentenbewegung gehörte. Der bis aufs Äußerste zugespitzte Konflikt der „Protestgeneration" 41 mit der NSTätergeneration, die sehr schnell nach 1945 wieder politische und gesellschaftliche Machtpositionen besetzt hatte, ist für das Verständnis der politisch-psychologischen Dynamik, die der Herausbildung des linken Antizionismus zugrunde lag, von zentraler Bedeutung. Der Antizionismus hatte politisch-psychologisch die Funktion eines „Massenmediums“42, in dem die widersprüchlichsten Wünsche, Projektionen und Identifikationen zusammengeführt werden konnten. Er gab Teilen der „Protestgeneration“ das gute Gefühl, auf der Seite der Schwachen und Unterdrückten zu stehen und die Menschenrechte $\mathrm{zu}$ verteidigen, sich von den im Schweigen und Verdrängen sich einkapselnden und sich selber als Opfer stilisierenden Eltern vehement abgrenzen und zugleich die von den Eltern übertragenen, unbewussten Schuld- und Schamgefühle in latenten oder auch sehr manifesten Aggressionen gegenüber den Juden ausleben zu können. ${ }^{43}$ Das antisemitische Ressentiment konnte in ein

${ }^{39}$ Siehe u. a. Martin Kloke, Israel und die deutsche Linke, Frankfurt a. M. 1994, S. 113ff.; Ders., Israel - Alptraum der deutschen Linken?, in: Brosch (Hrsg.), Exklusive Solidarität, S. 301-323, hier S. 303.

40 Siehe u. a. Lars Rensmann, Demokratie und Judenbild, S. 315; Broder, Der ewige Antisemit, S. 124ff.

41 „Die Studenten haben so ein wenig die Rolle der Juden übernommen“, formulierte Adorno in einer Erklärung aus Anlass des Todes des Studenten Benno Ohnesorg, der am Rande der Anti-Schah-Demonstration am 2. Juni 1967 von der Polizei erschossen wurde. Dass sich in dem Hass gegen die revoltierenden Studenten etwas von dem Hass gegen die Juden wiederholte, entging Adorno ebenso wenig wie das Scheinhafte der studentischen Revolte, dessen antisemitisches Potenzial er in einem Brief an Herbert Marcuse im Sommer 1969 deutlich benannte: „Nachdem man in Frankfurt den israelischen Botschafter niedergebrüllt hat, hilft die Versicherung, das sei nicht aus Antisemitismus geschehen [...], nicht das mindeste. [...] Du müsstest nur einmal in die manisch erstarrten Augen derer sehen, die, womöglich unter Berufung auf uns selbst, ihre Wut gegen uns kehren“. (Adorno zit. n. Wolfgang Kraushaar, Abspaltung und Potenzierung. Zum Verhältnis von Antizionismus und Antisemitismus in der militanten Linken der Bundesrepublik Deutschland, in: Brosch (Hrsg.), Exklusive Solidarität, S. 325-346, hier S. 331f.) - Die Verdienste der 68er-Bewegung für die Demokratisierung der politischen Kultur der Bundesrepublik stehen außer Frage. Hier geht es allein um die antisemitischen Tendenzen im linken Antizionismus und deren politisch-psychologischer Genese.

42 Adorno, Zur Bekämpfung des Antisemitismus, S. 366.

43 Auch die unbewusste Übertragung des antisemitischen Erbes an die zweite Generation ist psychoanalytisch mittlerweile gut erforscht. (Siehe u. a. Jan Lohl, Gefühlserbschaft und Rechtsextremismus. Eine sozialpsychologische 
antiimperialistisches Feindbild eingebaut und mit klassentheoretischen und kapitalismusbezogenen Freund-/Feindschemata verknüpft werden. Israel wurde so als „Speerspitze“ oder „Brückenkopf des Imperialismus“ imaginiert, als Zentrum der imperialistischen Weltverschwörung, das zusammen mit den USA die Welt beherrscht oder zu beherrschen anstrebt. Folgerichtig solidarisierten sich die antizionistischen Antiimperialisten mit dem als homogen und ethnisch vorgestellten ,palästinensischen Volk“ oder den „arabischen Völkern“, unterstellten dem phantasierten jüdischen Täterkollektiv eine besondere Nähe zur feindlichen Klasse, dem Kapitalismus und den zu bekämpfenden Ideologien des Rassismus, Nationalismus und Faschismus und taten alles, um die „unterdrückten Völker“ in ihrem bewaffneten Kampf gegen die „Zionisten“ und „Imperialisten“ zu unterstützen. ${ }^{44}$

Im Prozess der Antizionisierung eines großen Teils der radikalen Linken entstand nicht nur eine zunehmend enthemmte antisemitische Propaganda, sondern es kam auch zu einer Reihe von antisemitischen Gewalttaten, wie man sie bis dahin nur aus dem rechten Spektrum kannte. Schon 1969 schändeten radikale Linke Gedenkstätten, fast zeitgleich folgten der Versuch eines Bombenattentats auf das Jüdische Gemeindehaus in Westberlin und Agitationen gegen israelische Wissenschaftler mit Parolen wie „Schlagt die Zionisten tot - macht den Osten rot". ${ }^{45}$ Auch in den Jahren darauf kam es immer wieder zu linken Gewaltattacken gegen Jüdinnen und Juden, die ihren Höhepunkt in der „Selektion von Entebbe “46 im Jahr 1976 fanden. ${ }^{47}$ Der Auschwitzüberlebende Yitzhak David, der sich an Bord des entführten Flugzeugs befand, traf etwas Richtiges, als er den

Studie zur Generationengeschichte des Nationalsozialismus, Gießen 2010, S. 193ff.; Ders./Angela Moré (Hrsg.), Unbewusste Erbschaften des Nationalsozialismus. Psychoanalytische, sozialpsychologische und historische Studien, Gießen 2014.)

${ }_{44}$ Claudia Globisch hat die semantische Struktur des antizionistischen Antiimperialismus an Texten aus der radikalen Linken detailgenau analysiert. (Claudia Globisch, Radikaler Antisemitismus. Inklusions- und Exklusionssemantiken von links und rechts in Deutschland, Wiesbaden 2013, S. 257ff.)

45 Siehe u. a. Rensmann, Demokratie und Judenbild, S. 310ff; Thomas Haury, Der neue Antisemitismusstreit der deutschen Linken, in: Doron Rabinovici/Ulrich Speck/Natan Sznaider (Hrsg.), Neuer Antisemitismus? Eine globale Debatte, Frankfurt a. M. 2004, S. 143-167; Thomas Haury, Der Antizionismus der Neuen Linken in der BRD. Sekundärer Antisemitismus nach Auschwitz, in: Arbeitskreis Kritik des deutschen Antisemitismus (Hrsg.), Antisemitismus - die deutsche Normalität. Geschichte und Wirkungsweise des Vernichtungswahns, Freiburg i. Br. 2015, S. 217-229; Wolfgang Kraushaar, Abspaltung und Potenzierung; Wolfgang Kraushaar, „Wann endlich beginnt bei Euch der Kampf gegen die heilige Kuh Israel?", München 1970; Ders., Über die antisemitischen Wurzeln des deutschen Terrorismus, Hamburg 2013.

${ }^{46}$ Kraushaar, Abspaltung und Potenzierung, S. 343.

${ }^{47}$ Mit der Entführung des Air-France-Flug 139 von Tel Aviv nach Paris am 27. Juni 1976 wollte das „Kommando Che Guevara“ der „Volksfront für die Befreiung Palästinas“ (PFLP) unter der Führung des ehemaligen Frankfurter Soziologiestudenten Wilfried Böse die Freilassung von 53 „Freiheitskämpfern“ für die „palästinensische Sache“ erzwingen, darunter auch Gefangene der RAF und der Bewegung 2. Juni. Nach der Landung auf dem Flughafen Entebbe in Uganda nahmen die Entführer eine Selektion zwischen den jüdischen und nichtjüdischen Geiseln vor: Die 147 nichtjüdischen Geiseln wurden freigelassen, die 70 israelischen und 34 weitere jüdische Passagiere mussten an Bord bleiben: „Die Geiselnehmer drohten, das Flughafengebäude zu sprengen und alle Geiseln umzubringen, wenn ihrer Forderung nach Freilassung der inhaftierten ,Kampfgenossen' nicht nachgegeben würde“. (Kraushaar, Abspaltung und Potenzierung, S. 344.) In einer Kommandoaktion der israelischen Armee konnten viele Geiseln befreit werden, einige starben. 
deutschen Flugzeugentführer und ehemaligen Frankfurter Soziologiestudenten Böse mit dem Satz konfrontierte: „I was mistaken when I told my children that there is a different Germany. When I see what you and your friends are doing to women, children and the elderly, I see that nothing has changed in Germany".48 Tatsächlich schien der bundesdeutsche Linksterrorismus nahtlos an die Exekutionspraktiken der NSTätergeneration anzuknüpfen, nur dass der zu vernichtende „Jude“ nun „Zionist“ hieß, man selber sich nicht mehr Antisemit, sondern Antiimperialist nannte und die Identifikation mit dem als „Opfer der jüdischen Weltverschwörung“ imaginierten völkisch-deutschen Kollektiv durch die Identifikation des als „Opfer des Weltzionismus“ imaginierten heldenhaften völkisch-palästinensischen Kollektivs ersetzt wurde. Auf der unbewussten Ebene des Generationenverhältnisses aber stellen sich die Dinge komplizierter und widersprüchlicher dar. Wenn die psychoanalytischsozialpsychologische These zutrifft, dass die auf den NS folgende Generation mit den verdrängten Schuldgefühlen der Elterngeneration „unausweichlich, weil unbewusst“49 identifiziert war, dann liegt es nahe, dass es sich bei dem militanten deutschen Antizionismus auch um eine besonders verdrehte Form unbewusster Schuldentlastung und „Wiedergutmachung“ gehandelt haben muss. In der Solidarisierung mit den vermeintlichen Opfern der Opfer und deren Kampf gegen Israel holten die Nachgeborenen nach, was sie sich von ihren Eltern gegenüber dem NS gewünscht hätten. Zugleich musste es für sie psychisch enorm entlastend gewesen sein, dass im antizionistischen Weltbild die ehemaligen Opfer zu mindestens ebenso schlimmen Tätern geworden waren wie ihre Nazi-Eltern. Dass sie mit dieser Täter-OpferVerkehrung die antisemitische Erbschaft ihrer Eltern übernahmen, war ihnen selbst vermutlich am wenigsten bewusst.

Der linke Antizionismus hatte seine große Zeit in den 1970er-Jahren, in der es quasi keine linksradikale Gruppe gab, die nicht antizionistisch war. ${ }^{50}$ Sein Niedergang begann bereits in den 1980er-Jahren und dann forciert im Nachgang zum zweiten Golfkrieg im Jahr 1990/91. In heftigen innerlinken Debatten spaltete sich die radikale Linke in eine weiterhin scharf antiisraelische Szene und eine dezidiert prozionistische Strömung, in der die - sich selbst so nennenden - „Antideutschen“ besonders hervorstachen. Den weltweiten Kommunismus als messianisches Fernziel propagierend, zelebrierte diese Gruppierung für die Zeit auf dem Weg dorthin eine „Form der Israelbegeisterung, die sich mit den Maximalpositionen der israelischen Rechten deckt".51 Trotz seines Bedeutungsverlustes hat der antizionistische Antisemitismus von links Wirkungen bis

48 Yossi Melman, Setting the Record Straight: Entebbe was not Auschwitz, in: Haaretz, 8.7.2011.

${ }^{49}$ Quindeau, Schuldabwehr und nationale Identität, S. 163.

50 „In ihrem sogenannten Antizionismus war sich die ansonsten häufig zerstrittene Linke - maoistische K-Gruppen, moskautreue DKP, antiautoritäre Linke, antiimperialistische Dritte-Welt-Solidaritätsgruppen bis hin zu den terroristischen ,Gruppen des bewaffneten Kampfes’ - während der gesamten 1970er Jahre überaus einig“. (Haury, Der neue Antisemitismusstreit, S. 205; siehe auch Kloke, Israel und die deutsche Linke.)

${ }^{51}$ Kloke, Israel - Alptraum der deutschen Linken, S. 315. 
weit über die Grenzen des eigenen Milieus hinaus erzielt. Fast ein Drittel der deutschen Bevölkerung ist heute der Meinung, dass das, „was der Staat Israel heute mit den Palästinensern macht, im Prinzip auch nichts anderes ist als das, was die Nazis im Dritten Reich mit den Juden gemacht haben“. Und der Aussage „Israel führt einen Vernichtungskrieg gegen die Palästinenser" stimmen aktuell sogar 40 Prozent der Befragten zu. ${ }^{52}$

\section{Falsche Israelfreunde: der „ehrbare“ Antisemitismus von rechts seit 1989}

Im Zuge des Vereinigungsprozesses der beiden deutschen Staaten nach 1989 und noch einmal verstärkt nach den islamistischen Terroranschlägen vom 11. September 2001 lassen sich drei Entwicklungen beobachten, in denen sich der Zusammenhang von Schuldentlastung und sekundärem Antisemitismus in der Bundesrepublik Deutschland neu konstelliert: der Aufstieg des antimuslimischen Rassismus in der sogenannten Mitte der Gesellschaft, der - nur scheinbar paradox - zugleich eine sekundärantisemitische Funktion übernimmt; der taktische Proisraelismus der Neuen Rechten, die gleichzeitig aggressiv geschichtsrevisionistisch agiert, und das Wiedererstarken des linken Antizionismus im Rahmen der Anti-Globalisierungsbewegung und eines weltweiten Anti-Israel-Kampfes, der sich unter anderem in der sogenannten BDS-Kampagne organisiert.

An der ideologischen Struktur des linken Antizionismus, der mit der Zweiten Intifada Anfang der 2000er-Jahre wieder an Aufwind gewann, hat sich wenig verändert. Neu sind aber die Bündniskonstellationen, die es in den 1970er und 1980er-Jahren noch nicht gab. ${ }^{53} \mathrm{Da}$ auch islamistische Terrorgruppen wie der Islamische Dschihad oder die Hamas, die offen Sympathie für den NS äußern und vernichtungsantisemitische Propaganda betreiben, als Teil des antiimperialistischen Befreiungskampfes betrachtet werden, gelten sie den deutschen Antizionisten als willkommene Bündnispartner. ${ }^{54}$ Zugleich wird der Staat der Auschwitzüberlebenden als „Verbrecherstaat“ dämonisiert und mit dem Nationalsozialismus gleichgesetzt. Die tausendfach im Internet, auf AntiIsrael-Demonstrationen und im antizionistischen Propagandamaterial $\mathrm{zu}$ findende Gleichsetzung des Davidsterns mit dem NS-Hakenkreuz transformiert die alte antisemitische Vorstellung vom ,Juden“ als dem absolut Bösen in die Gegenwart. Als Motor antisemitischer Propaganda erweist sich dabei immer mehr die BDS-Kampagne gegen Israel. ${ }^{55}$

52 Bericht des Unabhängigen Expertenkreises Antisemitismus, 7.4.2017.

53 Siehe auch den Beitrag von Daniel Rickenbacher in diesem Band.

54 Haury, Der neue Antisemitismusstreit, S. 149f.

55 „Boycott, Divestment and Sanctions“ (abgekürzt BDS) ist eine weltweite propalästinensisch-antizionistische Kampagne, die Israel wirtschaftlich, kulturell und politisch isolieren und ruinieren will. 
Gibt es über den antizionistischen Teil der deutschen Linken kaum Neues zu berichten, so versuchen sich die Neuen Rechten nach 1989 verstärkt in der Etablierung eines „ehrbaren“ Antisemitismus eigener Art, der sie endlich wieder reputationsfähig machen soll. In maximaler Distanzierung vom NS stilisieren sie sich als die wahren Antifaschisten. Von der Wirmer-Flagge über den Stauffenberg-Kult bis hin zu IsraelSolidaritätsbekundungen auf Pegida-Demonstrationen - alles baut in der Neuen Rechten auf dem Mythos von den „konservativen Revolutionären“ auf, die zwischen 1933 und 1945 heldenhaften Widerstand gegen die Nazis geleistet hätten und deren Erben heute ebenso entschlossen dem „Islamo-Faschismus“ entgegenträten. Tatsächlich aber ist diese Rechte auch heute genau das, was sie schon immer war: nationalistisch und antisemitisch. Für die Neue Rechte nämlich sind - dies hat Volker Weiß in seinem Buch „,Die autoritäre Revolte“56 herausgearbeitet - das Muslim-Bashing wie auch die Israel-Solidarität nur taktische Mobilisierungsressourcen, inhaltlich aber sind sie Nebenschauplätze. Im Zentrum steht der „Ethnozid am deutschen Volk“. Dieser drohe nicht durch den Islam, sondern durch den ,großen Austausch“, wie es im neurechten Jargon heißt, also durch Einwanderung. Und hinter dieser stehe der eigentliche, der innere Feind. Um diesen Gedankengang zu verstehen, ist es notwendig, sich den großen Einfluss des - von Waldemar Gurian sarkastisch so genannten - „Kronjuristen des Dritten Reiches“, Carl Schmitt, im neurechten Denken klar zu machen. ${ }^{57}$ In Carl Schmitts „Theorie des Partisanen“58 wird zwischen ,wirklichem“ und ,absolutem Feind“ unterschieden. Der „wirkliche Feind“ ist sichtbar, berechenbar, der Krieg gegen ihn ist führbar, also auch kontrollierbar. Die ,absolute Feindschaft“ hingegen zielt auf die totale Vernichtung des Gegners. Genau damit haben wir es aus Sicht der Neuen Rechten heute zu tun. Die Vernichtung des „Eigenen“ beginne mit der „Amerikanisierung“ Westdeutschlands nach 1945, setze sich fort in der „Kulturzerstörung von 1968“ und gipfele im „linksliberalen Eine-Welt-Gender-Multikulti-Albtraum“ von heute. Hinter diesem Vernichtungswerk aber stehe das Prinzip universalistischer Nicht-Identität. Der Vordenker der Nouvelle Droite, Alain de Benoist, formulierte dies in einem Gespräch mit der neonazistischen Zeitschrift Hier \& Jetət im Jahr 2010 so: „Die größte Bedrohung unserer Identität ist keine andere Identität, sondern der politische Universalismus in allen seinen Formen, der die Volkskulturen und unterschiedlichen Lebensstile bedroht, und der sich anschickt, die Erde in einen homogenen Raum zu verwandeln. "59 Von hier aus wird auch klar, warum die Feindschaft gegen den Islam nur taktischer Art ist. Denn in der Logik des neurechten Ethnopluralismus sind alle Völker im Prinzip Waffenbrüder, auch das deutsche Volk und die arabischen Völker. Und so verbindet die völkischen Nationalisten Europas und die

56 Volker Weiß, Die autoritäre Revolte. Die Neue Rechte und der Untergang des Abendlandes, Stuttgart 2017.

${ }^{57}$ Siehe hierzu Samuel Salzborn, Angriff der Antidemokraten. Die völkische Rebellion der Neuen Rechten, WeinheimBasel 2017, S. 63ff.

58 Carl Schmitt, Theorie des Partisanen. Zwischenbemerkung zum Begriff des Politischen, Berlin 1963.

${ }^{59}$ „Auf den Trümmern des bürgerlichen Individualismus.“, Arne Schimmer, Interview mit Alain de Benoist, in: Hier おetz̧t 15 (2010), S. 26-35, hier S. 30f. 
islamischen Fundamentalisten von heute eine „Hassliebe“ (Volker Weiß), weil aus Sicht der Rechten beide ja letztlich nichts anderes wollen, als das Vernichtungswerk der Moderne aufzuhalten.

Von hier ist es dann nur noch ein kleiner Schritt, dem ,absoluten Feind“ einen Namen und eine Gestalt zu geben. Was bei den Neuen Rechten krypto-antisemitisch meistens versteckt bleibt und nur in Andeutungen sichtbar wird, wird in der alten Rechten offen ausgesprochen. Einen ,geistigen Giftpilz der Gemeinschaftszersetzung“ hat der NPDKader und Autor der „Deutschen Stimme“, Jürgen Gansel, den Philosophen des NichtIdentischen, Adorno, zu dessen 35. Todestag im Jahr 2004 genannt. ${ }^{60}$ Für ihn ist Adorno der Inbegriff ,des entwurzelten jüdischen Intellektuellen“. Und auch aktuell fehlt fast nie der Hinweis auf die „Kulturmarxisten“ von der „Frankfurter Schule“, wenn von der „Vergiftung des deutschen Volkes“ nach 1945 die Rede ist. Adorno steht dabei nur stellvertretend für den ,jüdischen Geist“ (Carl Schmitt), dem es aus Sicht der Neuen Rechten durch die Etablierung eines „Dauer-Schuldkults“ gelungen sei, die „deutsche Identität" fast völlig zu zerstören. Wendet man also den Blick von der Agitations- auf die Ideologieebene - dem Hauptfeld neurechten Schaffens -, so ist klar, dass der für die Rechte konstitutive Zusammenhang von Nationalismus und Antisemitismus sich keineswegs aufgelöst hat, wie manchmal behauptet wird. Ganz im Gegenteil: Er hat sich - und dies hat der von der alten wie der neuen Rechten gehasste Adorno schon früh sehr genau gesehen - nach Auschwitz sogar noch verschärft.

Völkischer Nationalismus lässt sich, so Adorno, sozialpsychologisch als kollektiver Narzissmus begreifen, der die Funktion einer Ersatzbefriedigung für die gesellschaftlich freigesetzten, zugleich aber unbefriedigt bleibenden narzisstischen Bedürfnisse vieler Einzelner hat. In den Masseninszenierungen des NS sei dieser kollektive Narzissmus ins Unermessliche gesteigert worden. Wurde die Niederlage Deutschlands von den völkischen Nationalisten als massive narzisstische Kränkung erfahren, so „lauert“ - wie Adorno es formuliert ${ }^{61}$ - ihr beschädigter kollektiver Narzissmus nur darauf, repariert zu werden. Was dies allerdings immer wieder verhindert, ist die mit Juden und dem Staat Israel verknüpfte Erinnerung an die eigene Verbrechensgeschichte. Juden werden im völkisch-nationalistischen Bewusstsein als entäußerte moralische Instanz und ewiger Störenfried der Erinnerung imaginiert und gehasst. Deshalb gilt, so Adorno: „Überall dort, wo man eine bestimmte Art des militanten und exzessiven Nationalismus predigt, wird der Antisemitismus gleichsam automatisch mitgeliefert".62 Genau dieser Zusammenhang lässt sich auch heute ohne große Mühe an vielen Äußerungen von AfDPolitikern, Pegida-Anhängern, Identitären und anderen Neurechten nachweisen, und zwar nicht nur, wenn die der NS-Propaganda entnommene Parole „Lügenpresse, halt die Fresse“ in die offen antisemitische ,Judenpresse, auf die Fresse“ umschlägt (wie auf einer

${ }^{60}$ Globisch, Radikaler Antisemitismus, A.1.2 Primärquellen.

61 Adorno, Was bedeutet: Aufarbeitung, S. 564.

62 Adorno, Zur Bekämpfung des Antisemitismus, S. 361. 
Pegida/Hogesa-Demonstration in Wuppertal im März 2015), sondern auch wenn davon Rede ist, dass Deutschland kein „souveränes Land“ sei, sondern die „Befehle aus Tel Aviv und Washington“ bekomme und die „USA von einer jüdischen Lobby“ regiert werde. ${ }^{63}$

\section{Zur sekundärantisemitischen Funktion des antimuslimischen Rassismus}

Bildet der auf die Restitution des Nationalstolzes ausgerichtete Geschichtsrevisionismus nach wie vor den Kern der Ideologiebildung der Rechten, so verstehen es ihre Agitatoren in geschickter Weise, durch Muslim-Bashing und Israel-Solidaritätsbekundungen Anschluss an den offiziellen Anti-Antisemitismus in der gesellschaftlichen Mitte zu finden. Sie beuten dabei das Entlastungsbedürfnis aus, das die öffentliche Anerkennung der Schuld sozialpsychologisch hervorbringt. Wie dies funktioniert, hat Ilka Quindeau in ihrer These eines modifizierten Sekundärantisemitismus skizziert. Quindeau sieht „die Leistung der [auf die NS-Tätergeneration nachfolgenden; Anm. WS] zweiten und dritten Generation in einer Anerkennung der Schuld [...], die in mühsamen, leidvollen kollektiven Prozessen einer ethisch-politischen Selbstverständigung errungen wurde“".64 Der psychologische Gewinn der Schuldanerkennung besteht zunächst darin, dass sie geradezu umkehrt zur schuldabwehrenden Tätergeneration - die Chance kollektiver narzisstischer Gratifikation eröffnet. Sie ermöglicht nämlich die kollektive Selbststilisierung als moralisch integre, demokratische Nation, der weltweit eine vorbildliche Auseinandersetzung mit ihrer Vergangenheit attestiert wird. Schuldbekenntnis und Erlösungserwartung, so Quindeau, gehen Hand in Hand und äußern sich in quasi religiösen Inszenierungen. Sie weist auf die Goldhagen-Debatte hin, in der so etwas wie eine Lust an der Schuld bei vielen Deutschen zu beobachten gewesen sei. ${ }^{65}$ Diese resultierte daraus, dass mit dem Bekenntnis zu einem spezifisch deutschen Vernichtungsantisemitismus das Versprechen auf Absolution einherging, das Goldhagen dann auch bereitwillig bediente. Das Selbstbild der geheilten, vom Antisemitismus

\footnotetext{
${ }^{63}$ Nachzuhören in der auf der ARD-Mediathek zugänglichen Panorama-Dokumentation „Pegida: Die Interviews in voller Länge“. (Siehe auch Salzborn, Angriff der Antidemokraten, S. $101 \mathrm{ff}$.) - Der von Julijana Ranc eingeführte Begriff der „Vergegenwärtigungsabwehr“ trifft ziemlich genau die Bewusstseins-/Unbewusstseinslage, die viele Anhänger von Pegida und AfD kennzeichnet: abgewehrt werden unlustvolle Gedanken und Gefühle, dabei handelt es nicht nur um „das Unbehagen am Holocaust und an der Schuld und dem Versagen der eigenen Eltern- und Großelterngeneration“, sondern auch um ,kulturell viel tiefer verankerte Gefühle wie etwa des Unheimlichen gegenüber Juden“. (Julijana Ranc, „Eventuell nichtgewollter Antisemitismus“. Zur Kommunikation antijüdischer Ressentiments unter deutschen Durchschnittsbürgern, Münster 2016, S. 161.)

${ }^{64}$ Quindeau, Schuldabwehr und nationale Identität, S. 164.

${ }^{65}$ Ebd., S. 163. - Lars Rensmann entgegnet aber zu Recht, dass mindestens ebenso so viel Schuldabwehraggression in den Reaktionen auf Goldhagens Buch zu beobachten war, was auch hier für eine Überlagerung verschiedener Formen des Sekundärantisemitismus spricht, mit der wir es heute zu tun haben könnten. (Rensmann, Demokratie und Judenbild, S. 336ff.)
} 
befreiten Nation aber gerät immer dann in Gefahr, wenn es zu manifester antisemitischer Gewalt kommt. Diese Gefahr kann nur durch eine rigorose Abspaltung gebannt werden. Genau hier bietet die Dramatisierung des migrationsbedingten Imports eines „muslimischen Antisemitismus“ einen Ausweg. Obwohl der größte Teil der antisemitischen Straftaten in Deutschland, wie die Statistiken zeigen, ${ }^{66}$ nach wie vor von völkischen Nationalisten verübt wird, wird in großen Teilen der medialen Öffentlichkeit die Hauptgefahr bei den „muslimischen Migranten“ verortet. Empirisch belegt ist dies nach wie vor kaum, ${ }^{67}$ aber sozialpsychologisch von großer Attraktivität. Indem der Antisemitismus auf ein Problem von „muslimischen Migranten“, zu denen kurzerhand dann auch die seit Generationen in Deutschland lebenden, ehemaligen türkischen Arbeitsmigranten und Arbeitsmigrantinnen gemacht werden, verkürzt wird, kann sich die „Wir"-Gruppe der Kinder und Enkel der ehemaligen NS-Volksgenossen in die Position moralischer Überlegenheit setzen und dadurch die Auseinandersetzung mit den eigenen antisemitischen Tendenzen vermeiden. Zugleich verknüpft die kulturalisierende, „religionisierende“ Wahrnehmung eines „muslimischen Antisemitismus“ den Kampf gegen eine angebliche Islamisierung Europas mit dem kategorischen Imperativ des „Nie wieder Auschwitz!“ ${ }^{68}$ In der patenten Formel vom „muslimischen Antisemitismus“ lässt sich ein kausaler Zusammenhang zwischen Islam und Antisemitismus behaupten. Antisemitismus wird zum Wesensmerkmal des Islam erklärt. Dadurch wird jeder Muslim und jede Muslima unter Antisemitismusverdacht gestellt. Sofern sie noch keine manifesten Antisemiten sind, sind sie es jedenfalls latent. Aber schlimmer noch: Die Muslime befinden sich in großer Zahl bereits in „unserem“ Land, und mit jedem neu ankommenden Muslim wächst die antisemitische Gefahr auch in Deutschland wieder. Historische Verantwortungsübernahme heißt dann - und hier liegt die Agitationschance, die die Neue Rechte nutzt -, sich der AfD als der einzigen politischen Kraft, die der Islamisierung und damit der Antisemitisierung Europas entschlossen entgegentritt, anzuschließen. ${ }^{69}$ Die Entsorgung des gesellschaftlichen Problems des Antisemitismus

66 Bericht des Unabhängigen Expertenkreises Antisemitismus, 10.11.2011, Deutscher Bundestag, Drucksache 17/7700, S. 14ff., [http://dipbt.bundestag.de/doc/btd/17/077/1707700.pdf], eingesehen 25.10.2018. - Selbstverständlich kann es sein, dass die vorhandenen Statistiken aufgrund mangelhafter oder einseitiger Erhebungstechniken die Realität verzerren und das Problem antisemitischer Gewalt, die von Muslimen ausgeht, unterschätzen, nur wäre auch dies zunächst einmal nachzuweisen.

${ }^{67}$ Auch die neueste Untersuchung von Günther Jikeli zum Antisemitismus bei Geflüchteten aus Syrien und dem Irak reicht dazu nicht aus. (Vergleich dazu den Beitrag Günther Jikelis in diesem Band, sowie Ders., Einstellungen von Geflüchteten aus Syrien und dem Irak zu Integration, Identität, Juden und Shoah. Forschungsbericht Dezember 2017, [https://ajcberlin.org/sites/default/files/ajc_studie_gefluechtete_und_antisemitismus_2017.pdf], eingesehen 25.10.2018; siehe auch Wolfram Stender, Konstellationen des Antisemitismus, in: Ders. u. a. (Hrsg.), Konstellationen des Antisemitismus. Antisemitismusforschung und sozialpädagogische Praxis, Wiesbaden 2010, S. 7-38, hier S. 19ff.) ${ }^{68}$ Dass die Reduzierung des Antisemitismus auf ein Importprodukt von „muslimischen Migranten“ sich schon längst in kulturrassistische Perspektiven verstrickt hat, zeigt sich z. B., wenn rechtspopulistische Initiativen wie „Pro Köln“ zur Gegenwehr gegen den ,importierten Antisemitismus“ aufrufen. (Alexander Häusler, Feindbild Moslem: Türöffner von Rechtsaußen hinein in die Mitte?, in: Gideon Botsch u. a. (Hrsg.), Islamophobie und Antisemitismus - ein umstrittener Vergleich, Berlin 2012, S. 169-190, hier S. 183f.)

${ }^{69}$ Exakt dieses Argumentationsmuster findet sich auch in den Gruppendiskussionen, die ein Forschungsteam der 
qua Projektion auf die von außen kommenden „Anderen“ lässt sich politischpsychologisch als Entlastungsstrategie interpretieren, die durch die migrationsgesellschaftlichen Entwicklungen möglich wird und die zugleich der von Quindeau analysierten Modifikation des sekundären Antisemitismus in der zweiten und dritten Generation nach Auschwitz entspricht. Der antimuslimische Rassismus gewinnt so, ohne dass dies den deutschen Islamfeinden bewusst sein muss, eine sekundärantisemitische Funktion. ${ }^{70}$

Das Fatale daran ist nicht nur die Verleugnung der antisemitischen Tendenzen in der Mehrheitsgesellschaft, sondern auch die Bagatellisierung des islamisierten modernen Antisemitismus durch die praktische Formel vom „muslimischen Antisemitismus“. Das Wissen von der gesellschaftlichen Transformation der traditionellen, religiösen Judenfeindschaft in den modernen Antisemitismus, ein Begleitprodukt der gesellschaftlichen Säkularisierung, gehört seit langem zum festen Bestandteil der Antisemitismusforschung. Die Reduzierung der islamisierten Variante des modernen Antisemitismus auf ein ausschließlich religiöses Phänomen aber ignoriert dies und abstrahiert damit auch von dessen spezifischer Vernichtungsqualität. Dies alles ist so gut erforscht und so bekannt, dass man auch bei den Verfechtern des Konstrukts „muslimischer Antisemitismus“ in der Wissenschaft den Eindruck nicht loswird, dass sie zum Zwecke eines billigen Muslim-Bashing die Bagatellisierung des modernen Antisemitismus wider besseren Wissens in Kauf nehmen. Statt sich dieser für Israel und alle Jüdinnen und Juden der Welt tödlich realen Gefahr zu stellen, was eine Auseinandersetzung mit der gesellschaftsgeschichtlichen Genese des islamisierten modernen Antisemitismus implizieren würde, gefällt man sich - im Wissenschaftsbetrieb nicht anders als in großen Teilen der Medien - in der Diffamierung einer Religion.

Der moderne Antisemitismus, der als Gegenbewegung zur politischen Emanzipation der Juden zwischen 1750 und 1850 entstand, ist zu einem globalen Phänomen geworden, das auch im einundzwanzigsten Jahrhundert eine geradezu unheimliche Aktualität hat. Sein Gravitationszentrum liegt heute in der arabischen Welt, seine Virulenz in Europa aber ist ungebrochen. Seine Besonderheiten in Deutschland wurden hier in groben Zügen skizziert. Antisemitismus lässt sich nicht erkennen, wenn man nur auf den Antisemitismus blickt. Notwendig ist die Erkenntnis der Gesellschaft, die ihn hervorbringt. Weder kann politische Psychologie kritische Gesellschaftstheorie ersetzen, noch ist sie ohne diese möglich. ${ }^{71}$ Eine politische Psychologie des Antisemitismus aber

Universität Göttingen mit „Pegidisten“ geführt hat. (Lars Geiges/Stine Marg/Franz Walter, Pegida. Die schmutzige Seite der Zivilgesellschaft, Bielefeld 2015, S. 125f.)

${ }^{70}$ Siehe u. a. Jan Lohl, „Ein total besiegtes Volk“. Tiefenhermeneutische Überlegungen zum Komplex „Geschichte, völkischer Nationalismus und Antisemitismus" im Rechtspopulismus, in: Meron Mendel/Astrid Messerschmidt (Hrsg.), Fragiler Konsens. Antisemitismuskritische Bildung in der Migrationsgesellschaft, Frankfurt a. M. 2017, S. 281303, hier S. 294ff.; Wolfram Stender, Ideologische Syndrome. Zur Aktualität des sekundären Antisemitismus in Deutschland, in: Markus Brunner u. a. (Hrsg.), Volksgemeinschaft, Täterschaft und Antisemitismus. Beiträge zur psychoanalytischen Sozialpsychologie des Nationalsozialismus und seiner Nachwirkungen, Gießen 2011, S. 240.

71 Zur gesellschaftskritischen Analyse des modernen Antisemitismus siehe u. a. Detlev Claussen, Grenzen der 
kann dabei helfen, über die unbewusste Tradierung antisemitischer Ressentiments in spezifischen politisch-kulturellen Kontexten aufzuklären. ${ }^{72}$ Für die Bekämpfung des Antisemitismus kann sie von großem Nutzen sein, folgt sie doch der Maxime, die Max Horkheimer bereits 1943 in einem Vortrag in der Jüdischen Gemeinde von Los Angeles formulierte: „Der Erfolg jedes Versuchs, den Antisemitismus zu bekämpfen, beruht weitgehend auf der Erkenntnis seiner verschiedenen Abarten, die im täglichen Leben oft nicht unterscheidbar sind. Wir müssen die soziale und psychologische Genese jeder einzelnen Variante erforschen “ ${ }^{73}$

Aufklärung. Die gesellschaftliche Genese des modernen Antisemitismus, Frankfurt a. M. 2005; Moishe Postone, Antisemitismus und Nationalsozialismus, in: Ders., Deutschland, die Linke und der Holocaust. Politische Interventionen, Freiburg i. Br. 2015, S. 165-194.

72 Grundlegend hierzu Rensmann, Demokratie und Judenbild, 2005; zum Verhältnis von Kritischer Theorie und kritischer politischer Psychologie siehe Alfred Krovoza/Christian Schneider, Politische Psychologie in der Bundesrepublik: Positionen und methodische Probleme, in: Helmut König (Hrsg.), Politische Psychologie heute (Leviathan Sonderheft 9/1988), Opladen 1988, S. 13-35.

73 Max Horkheimer, Zur Psychologie des Antisemitismus [1943], in: Ders., Gesammelte Schriften, Bd. 12, Frankfurt a. M. 1985, S. 172-183, hier S. 178. 